\title{
Detection of Steer Defecation Events using an Accelerometer
}

\author{
Nariyasu WATANABE ${ }^{1 *}$, Rena YOSHITOSHI ${ }^{1}$, Jihyun LIM $^{1}$, \\ Kensuke KAWAMURA ${ }^{2}$ and Seiichi SAKANOUE ${ }^{3}$ \\ ${ }^{1}$ Japanese Black Cattle Production and Wildlife Management Research Division, Western Region \\ Agricultural Research Center, National Agriculture and Food Research Organization, Oda, Japan \\ 2 Japan International Research Center for Agricultural Sciences, Tsukuba, Japan \\ ${ }^{3}$ Dairy Production Research Division, Hokkaido Agricultural Research Center, National Agriculture \\ and Food Research Organization, Sapporo, Japan
}

\begin{abstract}
Understanding the spatio-temporal elimination pattern of grazing cattle is important for grazing management. We thus developed a new method of detecting defecation events using a three-axis accelerometer. The accelerometer was fixed on the tails of three Japanese Black steers in a pasture, with the $x$-, $y$-, and $z$-axes being set to the front-to-back, side-to-side, and vertical directions relative to the normal tail position, respectively. The defecation behavior was also visually observed. The 3 -sec moving average was calculated from raw acceleration data and charted along the time course. The $x$-axis and $z$-axis accelerations showed convex upward and downward curves, respectively, at the defecation events. By using the synchronous signs of both curves, we could visually detect virtually all defecation events. And in order to detect defecation events automatically, we created six variables (i.e., maximum, minimum, and area in convex curve per $30 \mathrm{sec}$ for $x$ - and $z$-axes) and applied quadratic discriminant analysis (QDA) and a support vector machine (SVM). The critical success index values in QDA and the SVM were 0.8 and 0.98 , respectively, using the leave-one-out cross-validation method. We concluded that the use of an accelerometer on a steer's tail is effective in visually and statistically detecting defecation events.
\end{abstract}

Discipline: Animal Science

Additional key words: cattle, dung, elimination behavior, support vector machine, tail

\section{Introduction}

Excreta are a major nutrient source for plants in a grazing system (During and McNaught 1961; During and Weeda 1973). Potassium and phosphorus are excreted mainly in urine and feces, respectively, and nitrogen is excreted in significant proportions in both feces and urine (Haynes and Williams 1993). However, a certain percentage of grazing animals' excreta is eliminated on inaccessible or undesirable sites such as resting and watering places (Nakamura and Fukukawa 1973; Hirata et al. 2009; Auerswald et al. 2010), and the nutrients in that excreta cannot be reused on grazing land (Parfitt 1980; Selbie et al. 2015). To increase the circulation of such nutrients in a grazing system, a grazing layout and schedule are needed based on an understanding of the spatio-temporal elimination pattern of the grazing animals.

To date, most studies on the elimination behavior of domestic animals have been conducted by behavioral observation (e.g., Orr et al. 2012) or by simply counting the number of fresh dung pads or urine patches per area (e.g., MacLusky 1960; Moir et al. 2011). Although these methods are reasonably reliable for measuring the excreta distribution in pastures, they are rather labor-intensive and thus such research has been limited to short-term periods and/or small areas. In addition, difficult conditions such as darkness, observation from a distance, and observer fatigue may cause underestimations of the elimination behavior and excreta distribution.

A new system is thus needed to monitor elimination behavior as a replacement for such labor-intensive methods. Betteridge et al. (2010) developed an automatic recording system for urination events during the grazing period that uses a thermistor suspended below the animal's vulva to detect a rise in temperature at urination. Moreover, an automatic defecation recording system must also be developed to determine the actual situation of animal excreta in a grazing system. 
Following advances in micro-electromechanical systems technologies, animal behavior studies using a miniaturized accelerometer have undergone rapid development. Animal-attached accelerometers measure changes in the velocity of the body over time and can quantify fine-scale movements and body postures unlimited by visibility, observer bias, or the scale of space use (Brown et al. 2013). Ueda et al. (2011) and Yoshitoshi et al. (2013) fitted an accelerometry-based activity monitor on the neck of cattle and succeeded in distinguishing foraging activity from other activities. Watanabe et al. (2008) and Giovanetti et al. (2017a) measured under-jaw accelerations of cattle on a three-axis accelerometer, and discriminated eating, ruminating, and resting activities in a grazed pasture. De Passillé et al. (2010) and Ledgerwood et al. (2010) fitted a three-axis accelerometer on the leg and classified the gait patterns (galloping, trotting, and walking) of calves and the lying/standing behaviors of dairy cows, respectively. Fukasawa et al. (2018) measured the sleeping posture of cattle in lying positions by fitting a three-axis accelerometer on a halter on the middle occipital region. Mansbridge et al. (2018) and Barwick et al. (2018) classified the eating behaviors in sheep using a three-axis accelerometer worn on the ear. The findings above clearly show that an accelerometer is a powerful tool for distinguishing animal behaviors.

However, few studies have used an accelerometer to investigate the excretion behavior of livestock. Bueno et al. (1981) successfully determined the urination and defecation events of cows by recording the tail position using a chart recorder connected to an FM receiver that obtained signals from an FM transmitter fixed on the cow's back. A cow first raises her tail before defecating or urinating. In contrast, a steer raises his tail only before defecating (Brownlee 1950; Albright and Arave 1997; Aland et al. 2002). We hypothesized that an accelerometer fixed on a steer's tail could more easily and precisely record the tail movements, and thus detect the tail-raise movement prior to defecation. In the present study, we explored the potential for detecting defecation events by recording the acceleration of steers' tail movements in a grazed pasture.

\section{Materials and methods}

\section{Animals and study site}

The study was conducted in 2011 on three Japanese Black steers stocked in a pasture (8.1 ha) with seven Japanese Black cows and their calves. The ages and body weights of the steers in May 2011 were as follows: Steer 1, 37 months old and $604 \mathrm{~kg}$; Steer 2, 37 months old and $652 \mathrm{~kg}$; and Steer 3, 12 months old and $278 \mathrm{~kg}$. The stocked pasture was located at the Hokkaido Agricultural Research Center, National Agriculture and Food Research Organization (HARC/NARO) (42 $\left.59^{\prime} \mathrm{N}, 141^{\circ} 24^{\prime} \mathrm{E}\right)$, Sapporo, Japan, and consisted of a relatively flat section (3.1 ha, average slopes of $2.1^{\circ}$ ) and a sloped section (5.0 ha, $8.6^{\circ}$ ). The three steers, seven cows, and calves could move freely in both sections of the pasture during the stocking period from May to October 2011.

\section{Detection of defecation behavior using an accelerometer}

We used an accelerometer (Hitachi AirSense ${ }^{\mathrm{TM}}$ Entry Model 02 Plus, Hitachi WirelessInfo, Tokyo; $43 \times 35$ $\times 15 \mathrm{~mm}$, weighing $40 \mathrm{~g}$ ) equipped with an orthogonal three-axis acceleration sensor ranging from $-2 \mathrm{G}$ to $2 \mathrm{G}$, and a data logger that could record data at 0.05 -sec intervals for 14 days. The acceleration sensor recorded accelerations related to changes in movements (dynamic accelerations) and static acceleration (gravity). The accelerometer was wrapped in a vinyl bag for waterproofing and fixed on the upper part of the tail (approx. 12-24 cm below the anus) of each steer by an elastic adhesive bandage (ELATEX 4, Alcare, Tokyo) (Fig. 1).

The $x$-, $y$-, and $z$-axes of the fixed accelerometer were set to longitudinal (front-to-back), horizontal (sideto-side), and vertical directions relative to the normal tail position, respectively. In the normal position, the tail simply hangs naturally straight downward. The tail position is generally maintained in walking, grazing, feeding, and idling activities (Albright and Arave 1997). When a steer defecates, its tail is always raised. A 3- to 4-hr visual observation of behavior was conducted nine times in September and October 2011 during a variety of activity periods, such as grazing and resting periods. For each observation, one observer recorded the activities of the steers at 1-min intervals and the time of defecation events, unless they were at a distant place.

\section{Preprocessing of acceleration data}

We calculated the 3 -sec moving average of raw acceleration data on the $x$ - and $z$-axes to smooth the fluctuations, and charted the changes over 4-min sets of moving averages using Excel 2007 Visual Basic for applications (Microsoft, Tokyo). The $y$-axis data (lateral tail movements) were not analyzed in this study because it was thought that the $y$-axis data would not be involved in the tail-raise movement.

On the 3-sec moving average chart, we checked whether the defecation events identified by actual observation could be detected from the acceleration waveform.

To automatically detect the steers' defecation 

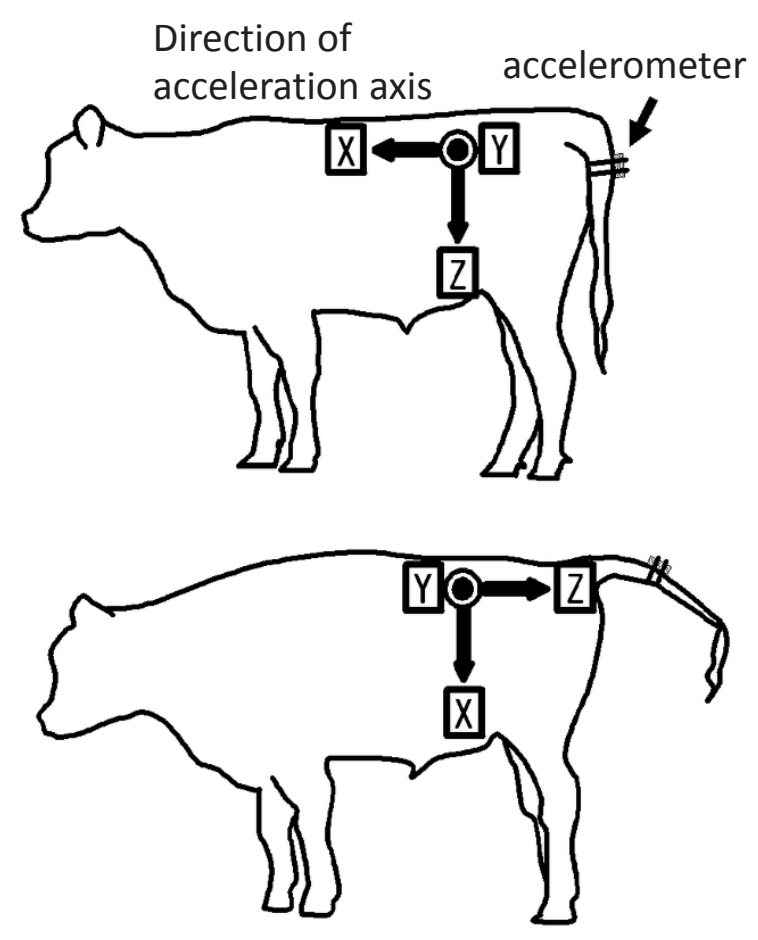

Fig. 1. The accelerometer attached to a steer's tail In the normal standing posture, where the tail simply hangs naturally straight downward, the $x$-, $y$-, and $z$-axes of the accelerometer indicate the longitudinal (front-to-back), horizontal (sideto-side), and vertical body axes, respectively (upper illustration). During defecation, the $x$-, $y$-, and $z$ - axes change directions close to the vertical, side-to-side, and front-to-back body axes, respectively (lower illustration).

events, we created the following six variables from the 3 -sec moving average of the acceleration data in a 30-sec moving window: the maximum, minimum, and area between the moving average data and minimum in the $x$-axis (or maximum in $z$-axis) per $30 \mathrm{sec}$ for the $x$-and $z$-axes, respectively.

$$
\begin{gathered}
\max x_{i}=\max \left\{x_{i+t} \mid t=0,1,2, \cdots, 30\right\} \\
\max z_{i}=\max \left\{z_{i+t} \mid t=0,1,2, \cdots, 30\right\} \\
\min x_{i}=\min \left\{x_{i+t} \mid t=0,1,2, \cdots, 30\right\} \\
\min z_{i}=\min \left\{z_{i+t} \mid t=0,1,2, \cdots, 30\right\} \\
\operatorname{area} x_{i}=\frac{1}{2} \sum_{t=1}^{30}\left[\left(x_{i+t}-\min x_{i}\right)+\left(x_{i+t-1}-\min x_{i}\right)\right] \\
\text { area } z_{i}=\frac{1}{2} \sum_{t=1}^{30}\left[\left(\max z_{i}-z_{i+t}\right)+\left(\max z_{i}-z_{i+t-1}\right)\right]
\end{gathered}
$$

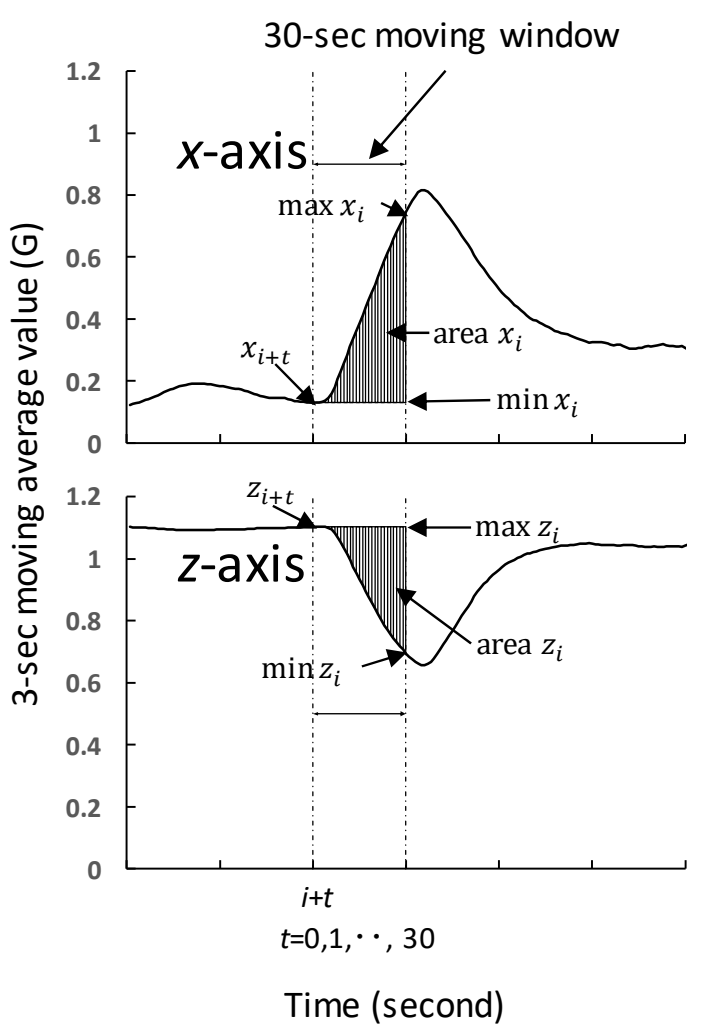

Fig. 2. An example of creation of the variables (max, min, area) to detect defecation events

Let $x_{i+t}$ and $z_{i+t}$ be the 3 -sec moving average values for the $x$ - and $z$-axes at the time $i+t$, respectively. A $30-\mathrm{sec}$ moving window is made in the range from $t=0 \mathrm{sec}$ (i.e., $i$ ) to $30 \mathrm{sec}$ (i.e., $i+30)$ at the time $i$. The maximum and minimum values in the window are regarded as max $x_{i}$ and $\min x_{i}$ in the upper figure and $\max z_{i}$ and $\min z_{i}$ in the lower figure. Area $x_{i}$ is calculated as the sum of 30 trapezoidal areas with the difference between $x_{i+t}$ and $\min x_{i}$ as the upper base, the difference between $x_{i+t-1}$ and $\min x_{i}$ as the lower base, and $1 \mathrm{sec}$ as the height, under the conditions: $1 \leq t \leq 30$. Area $z_{i}$ is calculated as the sum of 30 trapezoidal areas with the difference between $z_{i+t}$ and $\max z_{i}$ as the upper base, the difference between $z_{i+t-1}$ and $\max z_{i}$ as the lower base, and $1 \mathrm{sec}$ as the height, under the conditions: $1 \leq t \leq 30$.

where $x_{i+t}$ and $z_{i+t}$ are the 3 -sec moving average values in the $x$ - and $z$-axes, respectively, $i$ is the second number of the acceleration recording, and $t$ is the time in sec in a 30-sec moving window (i.e., 0-30) (Fig. 2). Area $x_{i}$ is the sum of 30 trapezoidal areas calculated at 1-sec intervals, where the difference between $x_{i+t}$ and min $x_{i}$ is regarded as the upper base, the difference between $x_{i+t-1}$ and min $x_{i}$ as the lower base, and $1 \mathrm{sec}$ as the height, under the conditions: $1 \leq t \leq 30$. Similarly, area $z_{i}$ is the sum of 30 trapezoidal areas regarding the difference between $z_{i+t}$ and $\max z_{i}$ as the upper base, the difference between $z_{i+t-1}$ and 
$\max z_{i}$ as the lower base, and $1 \mathrm{sec}$ as the height, under the conditions: $1 \leq t \leq 30$. These six variables were calculated every sec. To create the subsequent dataset as defecation data, we sampled one dataset of the six variables every time the tail began to rise for defecation, based on behavior observation data. As non-defecation data, we also sampled one dataset every minute when no defecation events but only maintenance activities were observed. A dataset was created for each steer by combining the sampled data (defecation and non-defecation data).

\section{Statistical analyses}

A Welch test was applied to determine whether the difference in means between the two events (defecation and non-defecation) for each variable in the dataset is significant (Rasch et al. 2011, Natori 2014). We conducted quadratic discriminant analysis (QDA) and used a support vector machine (SVM) with a Gaussian Radial basis kernel function to classify both events for each steer and for all three steers. Discriminant analysis (DA) is an effective method of classifying animal activities (Giovanetti et al. 2017b, Decandia et al. 2018). In particular, QDA can be used without the assumption of homogeneity in the variance-covariance matrix between activities (events), although the assumption of a normal distribution in each activity is needed (Mizuta et al. 2005, Jin 2007). A kernel SVM is also a powerful method of classifying animal activities with a linear approach, using a kernel function that transforms a dataset with a nonlinear data structure into a linearly separable dataset in higher-dimensional feature space (Jin 2007, Martiskainen et al. 2009).

The developed models were validated by the leaveone-out cross-validation $(\mathrm{CV})$ or leave-one-head-out $\mathrm{CV}$ method. In the leave-one-out $\mathrm{CV}$ method, the training model was created using all but one data on each steer or all three steers, and the model was validated using the excluded one as test data. The above method was repeated while assigning the test to all the data with replacement, and we compared the results obtained from the test data with the actual observed events. In the leave-one-head-out $\mathrm{CV}$ method, the model was created using the data on all the steers except one head, and the model was validated using the excluded steer's data (Tsenkova et al. 2009).

We evaluated the predictive accuracy obtained with QDA and the SVM by using the critical success index (CSI) values that range from 0 to 1 , with 0 indicating no correct answer and 1 indicating all correct answers (Schaefer 1990). The CSI focuses on the forecasts of rare events, that is, the ratio of the number of true positives (correct event forecasts, hits) to the number of true positives + false positives (incorrect event forecasts, false alarms) + false negatives (missed events, misses). True negatives (correct no-event forecasts) are ignored in this index to overcome the problem of numerous correct no-event forecasts (i.e., common maintenance behaviors-grazing, ruminating, resting without defecating) that increase the correct forecast rate.

The creation of the acceleration variables, Welch test, and subsequent QDA and SVM with CV were performed using statistical software "R" ver. 3.4.3 (R Core Team 2017). In QDA and the SVM, we used the "qda" function in the package "MASS" and the "ksvm" function in the package "kernlab" in " $\mathrm{R}$ " with the "leave-one-outcross-validation" argument or the "predict" function, respectively.

\section{Results}

\section{Behavior observations of defecation events}

Based on 72.3-hr observation, a total of 52 defecation events and their prior activities were recorded as listed in Table 1. The total numbers of observed defecation events for Steers 1, 2, and 3 were 18, 24, and 10, respectively. The activities observed just before the defecation events for all steers were grazing (29 times), standing from lying (11 times), walking (10 times), and resting (2 times).

\section{Visual detection of defecation events using acceleration change charts}

Figure 3 shows the 3 -sec moving average values in the $x$ - and $z$-axes in defecating and other activities. The $x$-axis values that indicate the front-back axial direction relative to the normal tail position showed a convex upward curve at the defecation events, whereas the $z$-axis

Table 1. Observation time and the number of recorded defecation events for three steers

\begin{tabular}{|c|c|c|c|c|c|c|}
\hline \multirow{2}{*}{ Steer no. } & \multirow{2}{*}{$\begin{array}{l}\text { Observation } \\
\text { time (hr) }\end{array}$} & \multirow{2}{*}{$\begin{array}{c}\text { No. of observed } \\
\text { defecation } \\
\text { events }\end{array}$} & \multicolumn{4}{|c|}{ Activities just before defecation events } \\
\hline & & & Grazing & $\begin{array}{l}\text { Standing } \\
\text { from lying }\end{array}$ & Walking & Resting \\
\hline 1 & 24.3 & 18 & 11 & 4 & 2 & 1 \\
\hline 2 & 23.8 & 24 & 13 & 4 & 7 & 0 \\
\hline 3 & 24.2 & 10 & 5 & 3 & 1 & 1 \\
\hline All heads & 72.3 & 52 & 29 & 11 & 10 & 2 \\
\hline
\end{tabular}



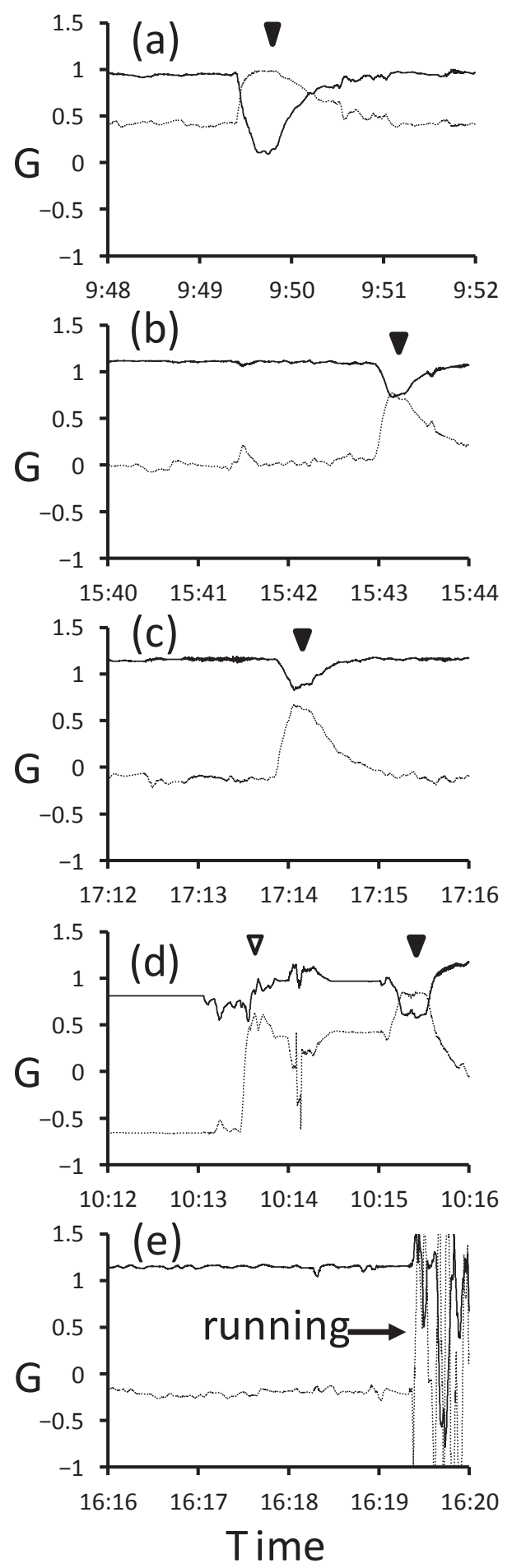

Fig. 3. Changes in the $x$-axis (dotted lines) and $z$-axis (solid lines) accelerations in defecating and other activities (a): Defecation event (30 September 2011 at 9:49) by Steer 1 during grazing. (b): Defecation (1 October 2011 at $15: 43)$ by Steer 2 during grazing. (c): Defecation (1 October 2011 at $17: 14$ ) by Steer 3 during walking. (d): Defecation ( 5 October 2011 at 10:15) by Steer 3 after standing from lying (at 10:13). (e): Running activity (5 October 2011 during 16:19-16:20) by Steer 3.

Black downward-pointing triangles: Defecation. White downward-pointing triangles: Standing events. values that indicate the vertical axial direction relative to the normal position showed a convex downward curve at the defecation events (Fig. 3 (a)-(c)). In most cases, the two curves intersected, although the enclosed area between both curves varied (Fig. 3 (a), (b)). In a few cases, the two curves did not intersect (Fig. 3 (c)).

When the steer rose (from lying to standing), the $x$-axis acceleration rose steeply, like a convex upward curve (Fig. 3 (d)). But due to the lower acceleration values of the $x$-axis while the steer was lying down, the movement from lying to standing could be distinguished from the movement of a defecation event. Moreover, tail-raise movements were also observed during running or walking (Fig. 3 (e)). However, almost all of these activities were not confused with defecation events, based on an examination of such differences as the peak height and width (sharpness). The signs of the synchronous convex upward and downward curves on the acceleration charts could be used as markers to detect defecation events.

Figure 4 shows an example of a $3-\mathrm{hr}$ period of acceleration changes on the tail. Here, three observed defecation events (at 10:17, 10:55, and 11:24) could be identified on the chart, although the defecation event at 10:17 just after standing was somewhat difficult to identify. Other tail movements were also observed on the charts, such as tapping movement of the tail while the steer was ruminating and lying down. However, due to the rapid acceleration changes (sharp peaks), those movements were never confused with defecation activities.

\section{Statistical classification of defecation events using acceleration variables}

In the observed defecation events, the means of the four acceleration variables $(\min x$, $\max x$, area $x$, and area $z$ ) were significantly higher than those in the nondefecation events; in contrast, the means of $\min z$ in the defecation events were significantly lower $(P<0.01$, Table $2)$. There were no significant differences in the means of $\max z$ between the two events. In a comparison between the steers, the means of variables in Steer 1 were higher in $\min x, \max x$, and area $z$, and lower in $\min z$ than those in Steers 2 and 3.

Using the five variables $(\min x, \max x$, area $x$, area $z$ and $\min z$ ) with significant differences, we applied QDA and the SVM for each steer and for all three steers. In QDA followed by leave-one-out CV (i.e., use of the same steer in training model and test sets), the CSI values for Steers 1, 2, 3, and all steers were 0.82, 0.92, 0.9, and 0.8, respectively (Table 3 ). In contrast, in QDA followed by leave-one-head-out CV (i.e., use of the different steers in training model and test sets), the CSI values for Steers 1, 2 , and 3 were $0.33,0.83$, and 0.91 , respectively. The low 
N. Watanabe et al.

Table 2. Statistical details of six variables $(\min x, \max x, \min z, \max z$, area $x$, and area $z)$ in defecation and nondefecation events, created from acceleration values on the tail of steers

\begin{tabular}{lccccccc}
\hline \hline & No. of data & Min $x$ & Max $x$ & Min $z$ & Max $z$ & Area $x$ & Area $z$ \\
\hline Steer 1 & & & & & & & \\
$\quad$ Defecation & 18 & $0.46 \pm 0.07$ & $0.98 \pm 0.02$ & $0.18 \pm 0.08$ & $0.94 \pm 0.04$ & $9.31 \pm 2.07$ & $11.53 \pm 2.79$ \\
Non-defecation & 1421 & $0.29 \pm 0.27$ & $0.37 \pm 0.29$ & $0.87 \pm 0.27$ & $0.93 \pm 0.25$ & $1.21 \pm 2.53$ & $0.75 \pm 1.37$ \\
$\quad$ Significance & & $P<0.01$ & $P<0.01$ & $P<0.01$ & NS & $P<0.01$ & $P<0.01$ \\
Steer 2 & & & & & & & \\
Defecation & 24 & $0.26 \pm 0.15$ & $0.85 \pm 0.06$ & $0.62 \pm 0.11$ & $1.04 \pm 0.09$ & $12.49 \pm 3.16$ & $7.67 \pm 2.03$ \\
Non-defecation & 1397 & $-0.15 \pm 0.25$ & $-0.07 \pm 0.30$ & $1.02 \pm 0.14$ & $1.06 \pm 0.10$ & $0.97 \pm 1.55$ & $0.38 \pm 0.64$ \\
Significance & & $P<0.01$ & $P<0.01$ & $P<0.01$ & NS & $P<0.01$ & $P<0.01$ \\
Steer 3 & & & & & & & \\
Defecation & 10 & $0.22 \pm 0.15$ & $0.81 \pm 0.08$ & $0.66 \pm 0.12$ & $1.08 \pm 0.09$ & $12.22 \pm 3.48$ & $7.42 \pm 2.22$ \\
Non-defecation & 1436 & $-0.30 \pm 0.24$ & $-0.19 \pm 0.33$ & $1.04 \pm 0.21$ & $1.09 \pm 0.16$ & $1.36 \pm 3.40$ & $0.55 \pm 1.41$ \\
Significance & & $P<0.01$ & $P<0.01$ & $P<0.01$ & NS & $P<0.01$ & $P<0.01$ \\
\hline
\end{tabular}

Means \pm standard deviation are shown. The significance of differences is determined by the Welch test.

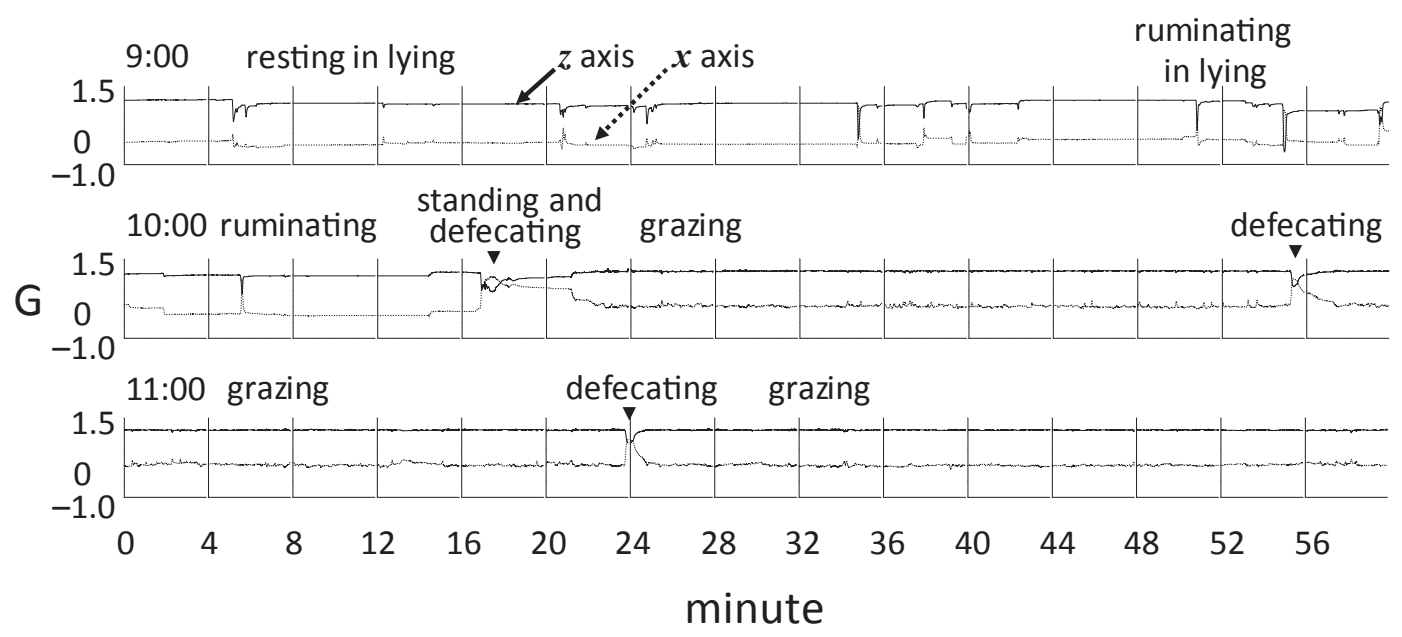

Fig. 4. An example of acceleration changes on the tail of Steer 2 over a 3-hr period on 5 October 2011, with behavioral observation

Three defecation events were observed at 10:17 (after standing), 10:55, and 11:24, the latter two of which occurred during grazing activity.

value for Steer 1 was due to many false alarms (FP). In the SVM followed by leave-one-out CV, the CSI values for Steers 1, 2, 3, and all steers were 0.95, 1, 1, and 0.98 , respectively, which are higher than those in QDA. However, in the SVM followed by leave-one-head-out $\mathrm{CV}$, the CSI values for Steers 1, 2, and 3 were $0,0.13$, and 0.3 , respectively, which are lower than those in QDA. The low values were due to many misses $(\mathrm{FN})$ in the detection of defecation events.

\section{Discussion}

\section{Visual detection of defecation events on acceleration charts}

Our present results confirmed that the use of an accelerometer on a steer's tail was effective in detecting defecation events. Such tail-raise movements are also observed during activities other than eliminating, such as galloping, gamboling, bucking, sexual activity, and calving (Bueno et al. 1981; Albright and Arave 1997). However, in the present study, almost all of the defecating events could be distinguished from other tail-raise movements by using the characteristic acceleration change sign (i.e., occurrence of synchronous convex upward and downward curves).

We consider the following generalizations regarding this acceleration change sign. When the steer raises its tail to defecate, the $x$-axis of the accelerometer, which initially indicates the front-back direction of the steer, changes from the front-back direction to the oblique or vertical direction. Then, when the tail is lowered after the end of defecation, the $x$-axis direction returns to the front- 
Table 3. Distribution of the prediction of defecation and non-defecation events and the critical success index (CSI) using quadratic discriminant analysis (QDA) and a support vector machine (SVM)

\begin{tabular}{|c|c|c|c|c|c|c|c|c|}
\hline Method & $\begin{array}{c}\text { Training } \\
\text { dataset }\end{array}$ & $\begin{array}{c}\text { Test } \\
\text { dataset }\end{array}$ & $\begin{array}{c}\text { No. of test } \\
\text { datat† }\end{array}$ & CSI & TP & FP & $\mathrm{FN}$ & $\mathrm{TN}$ \\
\hline \multirow[t]{7}{*}{ QDA } & Steer 1 & Steer $1 \dagger$ & $1,439(18)$ & 0.82 & 18 & 4 & 0 & 1,417 \\
\hline & Steer 2 & Steer $2 \dagger$ & $1,421(24)$ & 0.92 & 24 & 2 & 0 & 1,395 \\
\hline & Steer 3 & Steer $3 \dagger$ & $1,446(10)$ & 0.90 & 9 & 0 & 1 & 1,436 \\
\hline & All steers & All steers $\dagger$ & $4,306(52)$ & 0.80 & 48 & 8 & 4 & 4,246 \\
\hline & Steers $2 \& 3$ & Steer 1 & $1,439(18)$ & 0.33 & 15 & 27 & 3 & 1,394 \\
\hline & Steers $1 \& 3$ & Steer 2 & $1,421(24)$ & 0.83 & 20 & 0 & 4 & 1,397 \\
\hline & Steers $1 \& 2$ & Steer 3 & $1,446(10)$ & 0.91 & 10 & 1 & 0 & 1,435 \\
\hline \multirow[t]{7}{*}{ SVM } & Steer 1 & Steer $1 \uparrow$ & $1,439(18)$ & 0.95 & 18 & 1 & 0 & 1,420 \\
\hline & Steer 2 & Steer $2 \uparrow$ & $1,421(24)$ & 1.00 & 24 & 0 & 0 & 1,397 \\
\hline & Steer 3 & Steer $3 \dagger$ & $1,446(10)$ & 1.00 & 10 & 0 & 0 & 1,436 \\
\hline & All steers & All Steers $\dagger^{\dagger}$ & $4,306(52)$ & 0.98 & 52 & 1 & 0 & 4,253 \\
\hline & Steers $2 \& 3$ & Steer 1 & $1,439(18)$ & 0.00 & 0 & 0 & 18 & 1,421 \\
\hline & Steers $1 \& 3$ & Steer 2 & $1,421(24)$ & 0.13 & 3 & 0 & 21 & 1,397 \\
\hline & Steers $1 \& 2$ & Steer 3 & $1,446(10)$ & 0.30 & 3 & 0 & 7 & 1,436 \\
\hline
\end{tabular}

$\dagger$ : A leave-one-out cross-validation method is used.

$\dagger \uparrow$ : The numbers inside the parentheses indicate the number of defecation.

CSI: Critical success index, $\mathrm{TP} /(\mathrm{TP}+\mathrm{FP}+\mathrm{FN})$

TP: True positive (hits)

FP: False positive (false alarms)

FN: False negative (misses)

TN: True negative (correct no-events forecasts)

back direction. In the process of these directional changes, the static acceleration value of the $x$-axis rises to $>0.8$ $\mathrm{G}$ (max $x$ value) with upward tail movement, and then reverts to the initial state with downward tail movement. The increase and decrease in the static acceleration value make a convex upward curve with the dynamic movement of the tail.

In contrast, the static acceleration value of the $z$-axis, which initially indicates the vertical direction of the steer, falls from near $1 \mathrm{G}$ (max $z$ value) to $0.18-0.66 \mathrm{G}$ (min $z$ values) during upward tail movement and then is recovered during downward tail movement. Consequently, the acceleration changes of the $z$-axis show a convex downward curve for the defecation event. By these mechanisms, the synchronous convex upward and downward curves occur in defecating events. Conversely, in other tail-raise movements, the tail does not rise vertically from the normal position to the front-back (body-axis) direction (i.e., no simple movement of gravity acceleration between $x$ - and $z$-axes). Moreover, the tail's up-and-down movement is more rapid than that in defecating, showing a sharp peak rather than a convex curve. For these reasons, we consider that the other tail-raise movements do not show the occurrence of synchronous convex upward and downward curves, and that the synchronous curves act as markers only for eliminating events.

\section{Statistical detection of defecation events}

The statistical classification of defecation events by the SVM had higher CSI values than those by QDA in the leave-one-out CV method. The higher classification rate by the SVM is in agreement with other studies (Meyer et al. 2003, Garcia-Ruiz et al. 2013). QDA classifies data into two groups using a quadratic function in the dimensional plane determined by the number of input variables. In contrast, a kernel SVM creates an optimal higher-dimensional plane from the input variables, and in the plane, it determines the largest margin (the support vector), which linearly divides the data into two groups. With its creation of the higher-dimensional plane, the kernel SVM classifier allows for a better separation and classification of the data compared with other models (Garcia-Ruiz et al. 2013; Kurita).

However, in the leave-one-head-out CV method (in which the predicted steer's own data was not included in creating the training model), the CSI values obtained by the SVM were lower than those obtained by QDA, which might be due to the strong discrimination power of the SVM. The mean values of the variables used differed greatly between Steer 1 and Steers 2 or 3 (Table 2). 
The difference between the steers significantly affected creation of the training model and resulted in low CSI values, especially for Steer 1 . In general, there is a slight difference between individuals in the tail's up-and-down movement, and tail-up form in defecating events. In addition, in fixing an accelerometer on the tail, the location and angle of the device on the tail may differ slightly due to the difference in the shape (line) of the tail and the difficulty of fixing the device on the tail. Therefore, the differences between individuals and between fixation trials should be considered when creating a training model. The predicted individual's own data or the data from various individuals and fixation trials should be included in the training samples.

\section{Future studies}

In the present study, the statistical detection of defecation events of steers was successful with high accuracy. In the case of cows, however, there is still the problem of events being misinterpreted as urination activities. Cows are known to raise their tails not only for defecation but also for urination (Brownlee 1950). Thus, studies on the elimination activity of cows must discriminate between defecation and urination activities. In a trial using an accelerometer fixed on cows' tail, the acceleration pattern (synchronous convex upward and downward curves) in urination events was similar to that in defecation events, although the curve shape was slightly different (Watanabe et al., unpublished). In future studies, further development of the detection model is needed, including the discrimination between the defecation and urination of cows through detailed analyses of the shape of the acceleration curves.

\section{Conclusion}

We developed a new method of detecting steer defecation events that uses a three-axis accelerometer fixed on the tail, in which the $x$-axis and $z$-axis are set to the front-back and vertical directions relative to the normal tail position, respectively. In defecation events, the $x$-axis and $z$-axis accelerations showed convex upward and downward curves, respectively. By using the signs of the two synchronous curves on the acceleration chart, we were able to visually discriminate steer defecation events from other activities. In addition, the automatic classification of defecation events by QDA or the SVM succeeded with high CSI values. These results suggest that the tail-acceleration measurement method is effective in detecting defecation events and will contribute to studies of in-pasture nutrient cycling.

\section{Acknowledgements}

We wish to express our sincere appreciation to Ms. Shizue Nakajima of the HARC/NARO Daily Production Research Division for her assistance with the data analysis and field experiments, and to Mr. Kimiaki Ueda, Mr. Yukio Akazawa, and Mr. Mitsuharu Kato of the HARC/NARO Research Support Center for their assistance in the field experiments and cattle grazing management.

\section{References}

Aland, A. et al. (2002) Diurnal distribution of dairy cow defecation and urination. Appl. Anim. Behav. Sci., 78, 43-54.

Albright, J. L. \& Arave, C. W. (1997) The behaviour of cattle. CAB International, Wallingford, UK.

Auerswald, K. et al. (2010) Coupling of spatial and temporal pattern of cattle excreta patches on a low intensity pasture. Nutr. Cycl. Agroecosyst., 88, 275-288.

Barwick, J. et al. (2018) Categorising sheep activity using a triaxial accelerometer. Comput. Electron. Agric., 145, 289-297.

Betteridge, K. et al. (2010) Sensors for detecting and logging spatial distribution of urine patches of grazing female sheep and cattle. Comput. Electron. Agric., 73, 66-73.

Brown, D. D. et al. (2013) Observing the unwatchable through acceleration logging of animal behavior. Anim. Biotelem., $1,20$.

Brownlee, A. (1950) Studies in the behaviour of domestic cattle in Britain. Bull. Anim. Behav., 8, 11-20.

Bueno, L. et al. (1981) Detection of parturition in cow and mare by a useful warning system. Theriogenology, 16, 599-605.

Decandia, M. et al. (2018) The effect of different time epoch settings on the classification of sheep behaviour using triaxial accelerometry. Comput. Electron. Agric., 154, 112-119.

De Passillé, A. M. et al. (2010) Use of accelerometers to describe gait patterns in dairy calves. J. Dairy Sci., 93, 3287-3293.

During, G. \& McNaught, K. J. (1961) Effects of cow urine on growth of pasture and uptake of nutrients. NZ. J. Agr. Res., 4, 591-605.

During, G. \& Weeda, W. C. (1973) Some effects of cattle dung on soil properties, pasture production, and nutrient uptake. NZ. J. Agr. Res., 16, 423-430.

Fukasawa, M. et al. (2018) The use of accelerometer to measure sleeping posture of beef cows. Anim. Sci. J., 89, 488-493.

Garcia-Ruiz, F. et al. (2013) Comparison of two aerial imaging platforms for identification of Huanglongbing-infected citrus trees. Comput. Electron. Agric., 91, 106-115.

Giovanetti, V. et al. (2017a) Automatic classification of feeding behaviours in Sarda cattle using tri-axial accelerometry with different time epoch settings. In Precision Livestock Farming '17, eds. Berckmans, D. \& Keita, A., The 8th European Conference on Precision Farming, Nantes, France, 357-365.

Giovanetti, V. et al. (2017b) Automatic classification system for grazing, ruminating and resting behaviour of dairy sheep using a tri-axial accelerometer. Livest. Sci., 196, 42-48.

Haynes, R. J. \& Williams, P. H. (1993) Nutrient cycling and soil fertility in the grazed pasture ecosystem. Adv. Agron., 49, 119-199.

Hirata, M. et al. (2009) Deposition and decomposition of cattle 
dung in forest grazing in southern Kyushu, Japan. Ecol. Res., 24, 119-125.

Jin, M. (2007) Data Science by ' $R$ '. Morikita Publishing, Tokyo. [In Japanese].

Kurita, T.: Introduction to support vector machine [In Japanese]. https://home.hiroshima-u.ac.jp/tkurita/lecture/svm.pdf.

Ledgerwood, D. N. et al. (2010) Evaluation of data loggers, sampling intervals, and editing techniques for measuring the lying behavior of dairy cattle. J. Dairy Sci., 93, 5129-5139.

Maclusky, D. S. (1960) Some estimates of the areas of pasture fouled by the excreta of dairy cows. Grass Forage Sci., 15, 181-188.

Mansbridge, N. et al. (2018) Feature selection and comparison of machine learning algorithms in classification of grazing and rumination behaviour in sheep. Sensors, 18, 3532. doi: $10.3390 / \mathrm{s} 18103532$.

Martiskainen, P. et al. (2009) Cow behaviour pattern recognition using a three-dimensional accelerometer and support vector machines. Appl. Anim. Behav. Sci., 119, 32-38.

Meyer, D. et al. (2003) The support vector machine under test. Neurocomput., 55, 169-186.

Mizuta, M. et al. (2005) Introduction to data mining by S-PLUS. Morikita Publishing, Tokyo. [In Japanese].

Moir, J. L. et al. (2011) The spatial coverage of dairy cattle urine patches in an intensively grazed pasture system. J. Agr. Sci., 149, 473-485.

Nakamura, Y. \& Fukukawa, T. (1973) Decomposition of organic materials and soil fauna in pasture. 1. Distribution of cow dung pats. Bull. Natl. Grassl. Res. Inst., 5, 14-19.

Natori, M. (2014) The Mann-Whitney U test and tests of average values for unequal variances. Primate Res., 30, 173-185 [In Japanese].
Orr, R. J. et al. (2012) Defaecation and urination behaviour in beef cattle grazing semi-natural grassland. Appl. Anim. Behav. Sci., 139, 18-25.

Parfitt, R. L. (1980) A note on the losses from a phosphate cycle under grazed pasture. NZ. J. Exp. Agr., 8, 215-217.

Rasch, D. et al. (2011) The two-sample $t$-test: pre-testing its assumptions does not pay off. Stat. Papers, 52, 219-231.

$\mathrm{R}$ Core Team (2017) R: a language and environment for statistical computing. R Foundation for Statistical Computing, Vienna, Austria. https://www.R-project.org.

Schaefer, J. T. (1990) The critical success index as an indicator of warning skill. Weather Forecast., 5, 570-575.

Selbie, D. R. et al. (2015) The challenge of the urine patch for managing nitrogen in grazed pasture systems. Adv. Agron., 129, 229-292.

Tsenkova, R. et al. (2009) Near infrared spectroscopy using short wavelengths and leave-one-cow-out cross-validation for quantification of somatic cells in milk. J. Near Infrared Spec., 17, 345-351.

Ueda, Y. et al. (2011) The use of a physical activity monitor to estimate the eating time of cows in pasture. J. Dairy Sci., 94, 3498-3503.

Watanabe, N. et al. (2008) Development of an automatic classification system for eating, ruminating and resting behavior of cattle using an accelerometer. Grassl. Sci., 54, 231-237.

Yoshitoshi, R. et al. (2013) Distinguishing cattle foraging activities using an accelerometry-based activity monitor. Range. Ecol. Manage., 66, 382-386. 